\title{
High-Frequency Material-Dependent Scattering Processes for Tilted Truncated Cylindrical and Disk-Shaped Targets
}

\author{
Philip L. Marston \\ Department of Physics, Washington State University, Pullman, WA 99164-2814
}

\begin{abstract}
When evaluating potential applications of high-frequency scattering by targets, it is important to be able to anticipate which features of the scattering may be attributed to material properties of the target and which features would also be present for rigid targets. These concerns may be especially important for the classification of completely exposed and partially buried targets. The overview here emphasizes previously published relevant experimental and theoretical results for backscattering by bluntly-truncated targets in water.
\end{abstract}

\section{INTRODUCTION}

This survey is concerned with the scattering of sound in water by truncated objects when the size significantly exceeds the acoustic wavelength. Diffraction from the edges of the object similar to the diffraction from a rigid object can be important for some orientations of the target. For certain viewing angles, however, elastic contributions to the scattering, can be much larger in magnitude than simple edge diffraction. Such elastic contributions can greatly enhance the visibility of truncated tilted cylinders imaged with a high frequency sonar [1]. There has been significant progress in understanding such enhancements with quantitative ray theory. In most of the cases reviewed here, distinct signatures are present which depend on the material properties of the target. In most of the cases noted the signatures depend on guided wave reflection properties at truncations. Examples of such truncations include the end of a cylinder or the rim of a tilted circular disk. In most of the cases noted, at the optimum tilt condition the enhancements have only a weak dependence on frequency and the enhancements are not dependent on the presence of a global normal-mode or "resonance" of the target. That is because the high-frequency target normal-modes are often significantly damped and the strong coupling of elastic guided waves with the acoustic field is such that local coupling processes can affect the scattering. Those local processes are often describable by ray theory.

To describe the general magnitude of the scattering, in the case of finite circular cylinders it is convenient to introduce the following normalization for a dimensionless form function $f$ of which is related to the far-field scattered pressure as follows:

$$
\mathrm{p}_{\text {scattered }}=\mathrm{p}_{\text {incident }}(a / 2 \mathrm{r}) f \exp (\mathrm{ikr}),
$$


where $a$ is the radius of the cylinder and $\mathrm{r}$ is the distance from the cylinder and the time convention $\exp (-\mathrm{i} \omega \mathrm{t})$ is used. With this normalization, if the cylinder is replaced by a fixed-rigid sphere having the same radius, then when $\mathrm{k} a>1$, $\left|\mathrm{p}_{\text {scattered }}\right|$ is such that $|f|$ is close to unity except in the case of near-forward scattering.

\section{Bluntly-Truncated Rigid Cylinders}

Prior to examining elastic scattering enhancements it is appropriate to consider the scattering by a perfectly rigid circular cylinder having flat ends. At high frequencies, there are significant enhancements in the directions of specular reflections from the sides and ends of such a target. For the case of backscattering, these enhancements are for broadside and end-on illumination. Elsewhere, however, at high frequencies the scattering is dominated by the interference pattern of the diffraction effects of the ends. In terms of the normalized form function used in Eq. (1), the magnitude of the end diffraction is of order $(\mathrm{k} a)^{-1 / 2}$ and $|f|$ is much less than unity when $\mathrm{k} a$ is large. See for example [2] and Section 2.12 of [3]. If $\mathrm{k} a$ is large and the ends of the cylinder are rounded, as is the case of a cylinder having hemispherical end-caps, then for backscattering by a tilted rigid cylinder, $|f|$ can be relatively close to unity.

\section{MERIDIONAL AND HELICAL RAY BACKSCATTERING ENHANCEMENTS}

An important feature of the backscattering enhancements visible in the sonar images in [1] is that the enhancements were limited to specific ranges of tilt angles of the cylinder. It was correctly hypothesized in [1] that the observed enhancements were associated with elastic excitations of the cylinder (supersonic or "leaky" Lamb waves) described by rays that travel down the meridian of the cylinder and reflect off the end. The relevant meridian is in the plane containing the incident wave vector and the cylinder's axis. Since this type of scattering contribution was outside the scope of previous quantitative ray theories for scattering by shells (as well outside the scope of computational studies available at that time) Marston et al. [4-7] extended quantitative ray theory for cylinders to include this type of backscattering enhancement. During the development of the ray theory, it turned out to be useful to test ray theory for the related case of scattering by tilted infinitely-long solid cylinders [4] and cylindrical shells [8].

\section{Solid Cylinders: Meridional, Helical and Face-Crossing Rays}

The first quantitative experimental test of the aforementioned theory concerned the backscattering by a bluntly truncated solid stainless-steel cylinder where (in the frequency range of interest) the relevant leaky wave was analogous to a Rayleigh wave on a flat surface [5]. The magnitude of the meridional peak was $|f| \approx 2.5$, but elsewhere typically $|f| \approx 0.2$. The theory (see [4] and Appendix A of [5]) described the general magnitude and width of the peak, though the observed peak was offset from the predicted location for far-field scattering. That offset was caused by the limited size of the facility used in the measurements. In modeling the scattering, it was necessary to approximate 
the reflection coefficient of a Rayleigh wave from the end of the cylinder. In related work, backscattering enhancements were also observed that were associated with helical rays [5,9] and rays crossing the flat face of the ends of the cylinder [9]. The interference pattern associated with diffraction from the edges at the ends of the cylinder was also visible but was generally weaker than the aforementioned enhancements [9].

\section{Meridional Ray Enhancements for Tilted Cylindrical Shells}

Computations [6-8] show that at high frequencies, the relevant meridional rays for shells are typically associated with the lowest antisymmetric and symmetric generalizations of Lamb waves commonly designated respectively as $a_{0}$ and $\mathrm{s}_{0}$ waves. Of these waves, the $a_{0}$ wave has a phase velocity that depends significantly on frequency so that the tilt condition for the enhancement depends on frequency. This was confirmed by measuring the backscattering as a function of frequency over a wide range of scattering angles $[6,10]$. The $a_{0}$ wave studied was on the branch that is important above the coincidence frequency of the shell. The coupling conditions were also confirmed by extending Rumerman's method [11] of approximating the backscattering by truncated thin shells (based on partial-waves) to the situation here where thin-shell assumptions were no longer applicable [5]. Subsequently qualitative [10] as well as quantitative $[7,12]$ experiments demonstrated that in certain frequency ranges the simply-supported boundary condition in Rumerman's partial-wave approximation needed to be modified. These end corrections are especially significant for the $a_{0}$ wave when the threshold frequency of the $a_{1}$ mode is approached [7,10,12]. The cut-off $a_{1}$ mode is associated with a nonpropagating flexure of the shell localized near the ends of the shell. The nonpropagating mode is analogous to the "nonpropagating near-field deflection" of an elastic beam discussed by Junger and Feit [13]. This can be demonstrated by noting that for plates in a vacuum, the complex dispersion relation of the cut-off branch of the $a_{1}$ mode of a plate wraps around (with an imaginary wavenumber) and at low frequencies becomes the nonpropagating flexural branch of the fourth-order plate dispersion relation. See for example Fig. 19 of Mindlin [14]. In the present case of a fluid-loaded shell the cut-off $a_{1}$ mode causes an extra radiation of sound near the ends of the shell which (from energy conservation) reduces the magnitude of the reflection coefficient of the propagating $a_{0}$ wave [7]. (This reduction of the reflection coefficient associated with fluid-loading at a truncation could at least in principle be described by hybrid FEM-BEM computational methods.) Quantitative ray theory described the general magnitude and tilt-conditions of the backscattering enhancements. With the normalization used in Eq. (1), the backscattering peak typically had $|f| \approx 6$ and an angular width given by theory $[7,12]$. The transient responses in the time-domain and time-frequency domains were also investigated $[6,12,15]$.

\section{Helical Ray Backscattering Enhancements for Tilted Cylindrical Shells}

Away from the meridional ray coupling condition, qualitative measurements demonstrated that helical ray contributions can be significant $[6,10,12,15]$. A ray model for these enhancements was developed and confirmed with quantitative measurements of 
the backscattering of tone bursts [16] and the case of a water-filled shell was investigated [17]. Helical waves were also computed and modeled in the infinite shell case [18].

\section{SCATTERING ENHANCEMENTS FOR FLAT ELASTIC AND PLASTIC CIRULAR DISKS}

For certain tilt conditions, experiments by Hefner and Marston [19,20] demonstrated enhanced backscattering caused by leaky Lamb wave launched on an elastic disk. Acoustic holography was used to verify the identity of the waves associated with the enhancements [19-21] and a quantitative ray theory was developed that gives a useful approximation of the magnitude [21]. These enhancements are superposed on the edge diffraction contributions for the disks. The specular and edge diffraction contributions were usually weaker except when the incident wave vector was nearly perpendicular to the face of the disk. For plastic disks the shear wave velocity was less than the speed of sound in water and different types of scattering enhancements were observed [21,22].

\section{ENHANCED BACKSCATTERING BY A TILTED ELASTIC CUBE}

A leaky wave mechanism exists for producing a locally flat backscattered wavefront from a tilted elastic cube [23]. Only one of the cube's three Euler angles is constrained to lie in a narrow range. Consequently, for a randomly oriented cube (or certain other square-cornered objects) this mechanism becomes the most likely cause of large high frequency backscattering. Calculations based on ray theory predict enhanced backscattering. Measurements confirmed the general magnitude of the prediction in the case of an appropriately tilted stainless steel cube.

\section{CYLINDERS FILLED WITH LOW SHEAR VELOCITY MATERIALS}

For some cases of interest, it is anticipated that thin-walled cylindrical shells may be filled with materials having shear wave velocities less than the speed of sound in the surrounding water or sediment. To gain insight, the scattering was measured and modeled for bluntly truncated solid plastic and rubber cylinders in water [24]. For a range of tilt angles a significant backscattering enhancement was anticipated and was observed. That enhancement is associated with a caustic that is partially related to the acoustic "rainbow" of the tilted cylinder. Typical peak values of $|f|$ exceeded 2.5 and the enhancement was evident for ka as small as 10. A ray theory for this enhancement was confirmed [24] and aspects of the theory were also confirmed with a light scattering experiment [25].

\section{Leaky Waves and Internal Loading of Shells by Plastics}

For some cylinders of interest, the attenuation rate of meridional leaky or helical waves on cylinders will be affected by the presence of internal loading by plastic-like

materials. Recent calculations by Marston suggest, however, that conditions can be 
found where the enhanced attenuation caused by placing a solid plastic cylinder inside a metal shell will not be sufficient to eliminate the aforementioned meridional ray enhancements.

\section{Low-Frequency Modes of Water-Filled Cylinders Having Open Ends}

Experiments with low-frequency modes of water-filled shells suggest that scattering enhancements associated with low-frequency internal length-wise modes can be significant [26]. An approximate theory was developed [27] based on the application of the generalized optical theorem [28]. One potential application in underwater acoustics is that such open-ended water-filled cylinders could be used as test targets having a simple spectrum of resonances.

\section{ACKNOWLEDGMENTS}

The Office of Naval Research supported this research.

\section{REFERENCES}

1. Kaduchak, G., Wassmuth, C. M., and Loeffler, C. M., "Elastic wave contributions in high resolution acoustic images of fluid filled, finite cylindrical shells in water," J. Acoust. Soc. Am. 100, 64-71 (1996).

2. Ufimtsev, P. Y., "Theory of acoustical edge waves," J. Acoust. Soc. Am. 86, 463-474 (1989).

3. Marston, P. L., "Geometrical and catastrophe optics methods in scattering," in Physical Acoustics, edited by R. N. Thurston and A. D. Pierce (Academic, Boston, 1992), Vol. 21, pp. 1-234.

4. Marston, P. L., "Approximate meridional leaky ray amplitudes for tilted cylinders: End-backscattering enhancements and comparisons with exact theory for infinite solid cylinders," J. Acoust. Soc. Am. 102, 358-369 (1997).

5. Gipson, K., and Marston, P. L., "Backscattering enhancements due to reflection of meridional leaky Rayleigh waves at the blunt truncation of a tilted solid cylinder in water: Observations and theory," $J$. Acoust. Soc. Am. 106, 1673-1680 (1999).

6. Morse, S. F., Marston, P. L., and Kaduchak, G., "High frequency backscattering enhancements by thick finite cylindrical shells in water at oblique incidence: experiments, interpretation and calculations," $J$. Acoust. Soc. Am. 103, 785-794 (1998).

7. Morse, S. F., and Marston, P. L., "Meridional ray backscattering enhancements for empty truncated tilted cylindrical shells: Measurements, ray model and effects of a mode threshold," J. Acoust. Soc. Am. 112, 1318-1326 (2002).

8. Morse, S. F., and Marston, P. L., "Meridional ray contributions to scattering by tilted cylindrical shells above the coincidence frequency: Ray theory and computations," J. Acoust. Soc. Am. 106, 2595-2600 (1999).

9. Gipson, K. and Marston, P. L. , "Backscattering enhancements from Rayleigh waves on the flat face of a tilted solid cylinder in water," J. Acoust. Soc. Am. 107, 112-117 (2000).

10. Morse, S. F., and Marston, P. L., "Degradation of meridional ray backscattering enhancements for tilted cylinders by mode conversion: Wide-band observations using a chirped PVDF sheet source," IEEE J. Ocean. Eng. 26, 152-155 (2001).

11. Rumerman, M. L., "Contribution of membrane wave reradiation to scattering from finite cylindrical steel shells in water," J. Acoust. Soc. Am. 93, 55-65 (1993).

12. Morse, S. F., "High Frequency Acoustic Backscattering Enhancements for Finite Cylindrical Shells in Water at Oblique Incidence," (Ph.D. diss., Washington State University, Pullman, WA, 1998).

13. Junger, M., and Feit, D., Sound, Structures, and Their Interaction, 2nd ed. (American Institute of Physics, Woodbury, 1993) p. 206. 
14. Mindlin, R. D., "Waves and vibrations in isotropic, elastic plates," in Structural Mechanics, Proceedings of the First Symposium on Naval Structural Mechanics (Pergamon, Oxford, 1960).

15 Morse, S. F., and Marston, P. L., "Backscattering of transients by tilted truncated cylindrical shells: time-frequency identification of ray contributions from measurements," J. Acoust. Soc. Am. 111, 12891294 (2002).

16. Blonigen, F. J. and Marston, P. L., "Leaky helical flexural wave backscattering contributions from tilted cylindrical shells in water: Observations and modeling," J. Acoust. Soc. Am. 112, 528-536 (2002).

17. Blonigen, F. J. and Marston, P. L., "Leaky helical flexural wave backscattering contributions from tilted water-filled cylindrical shells," J. Acoust. Soc. Am. 113, 309-312 (2003).

18 Blonigen, F. J. and Marston, P. L., "Leaky helical flexural wave scattering contributions from tilted cylindrical shells: Ray theory and wave-vector anisotropy," J. Acoust. Soc. Am. 110, 1764-1769 (2001).

19. Hefner, B. T. and Marston, P. L., "Backscattering enhancements associated with the excitation of symmetric Lamb waves on a circular plate: direct and holographic observations," Acoustics Research Letters Online 2, 55-60 (2001) \{http://ojps.aip.org/ARLO/top.html\}.

20. Hefner, B. T., and Marston, P. L., "Backscattering enhancements associated with antisymmetric Lamb waves confined to the edge of a circular plate: direct and holographic observations," Acoustics Research Letters Online 3, 101-106 (2002) \{http://ojps.aip.org/ARLO/top.html\}.

21. Hefner, B. T., "Acoustic Backscattering Enhancements for Circular Elastic Plates and Acrylic Targets, the Application of Acoustic Holography to the Study of Scattering from Planar Elastic Objects, and Other Research on the Radiation of Sound" (Ph.D. diss., Washington State University, Pullman, WA, 2000).

22. Hefner, B. T., and Marston, P. L., "Acoustic backscattering and coupling processes for elastic and plastic circular disks in water: Direct and holographic observations," J. Acoust. Soc. Am. 109, 2489 (A) (2001).

23. Gipson, K., and Marston, P. L., "Backscattering enhancements due to retroreflection of ultrasonic leaky Rayleigh waves at corners of solid elastic cubes in water," J. Acoust. Soc. Am. 105, 700-710 (1999).

24. Blonigen, F. J., and Marston, P. L., "Backscattering enhancements for tilted solid plastic cylinders in water due to the caustic merging transition: Observations and theory," J. Acoust. Soc. Am. 107, 689-698 (2000).

25. Marston, P. L., Zhang, Y. B., and Thiessen, D. B., "Observation of the enhanced backscattering of light by the end of a tilted dielectric cylinder owing to the caustic merging transition," Appl. Opt. 42, 412-417 (2003).

26. Osterhoudt, C. F., and Marston, P. L., "Tilt angle dependence of backscattering enhancements from organ pipe modes of open water-filled cylinders: Measurements and models," J. Acoust. Soc. Am. 113, 2334 (A) (2003)

27. Marston, P. L., and Osterhoudt, C. F., "Modeling scattering enhancements at isolated resonances using energy conservation, reciprocity, symmetry, and the optical theorem," J. Acoust. Soc. Am. 113, 22842285 (A) (2003)

28. Marston, P. L., "Generalized optical theorem for scatterers having inversion symmetry: Applications to acoustic backscattering," J. Acoust. Soc. Am. 109, 1291-1295 (2001). 